\title{
Índices antropométricos e retinolemia em crianças menores de cinco anos do e Estado da Paraíba
}

\section{(1)}

\author{
Anthropometric indices and serum retinol \\ levels of children under age five years \\ from the State of Paraíba, Brazil
}

Daiane de QUEIROZ1

Adriana de Azevedo PAIVA²

Jacqueline Santos da Fonsêca Almeida GAMA ${ }^{3}$

Zilka Nanes LIMA ${ }^{4}$

Dixis FIGUEROA PEDRAZA 5

RES U M O

\section{Objetivo}

Avaliar a relação entre os índices antropométricos e os níveis de retinol sérico em crianças menores de cinco anos do Estado da Paraíba.

\section{Métodos}

Estudo de corte transversal, de base populacional, envolvendo 1205 crianças. O estado nutricional de vitamina A foi avaliado pelas concentrações séricas de retinol. O estado nutricional antropométrico foi avaliado utilizando-se os índices peso/altura e altura/idade.

\section{Resultados}

Crianças na faixa etária de 6 a 12 meses com deficit de altura apresentaram concentrações de retinol sérico significantemente inferiores quando comparadas às crianças sem deficit de altura $(p=0,02)$.

\footnotetext{
${ }^{1}$ Universidade Estadual da Paraíba, Programa de Pós-Graduação em Saúde Pública. Campina Grande, PB, Brasil.

2 Universidade Federal do Piauí, Departamento de Nutrição, Programa de Pós-Graduação em Alimentos e Nutrição. Teresina, PI, Brasil.

${ }^{3}$ Universidade Estadual da Paraíba, Departamento de Enfermagem, Curso de Enfermagem. Campina Grande, PB, Brasil.

${ }^{4}$ Universidade Estadual da Paraíba, Departamento de Farmácia, Laboratório de Análises Clínica. Campina Grande, PB, Brasil.

5 Universidade Estadual da Paraíba, Departamento de Enfermagem, Programa de Pós-Graduação em Saúde Pública. Av. das Baraúnas, 351, Bodocongó, 58429-500, Campina Grande, PB, Brasil. Correspondência para/Correspondence to: D FIGUEROA PEDRAZA. E-mail: <dixisfigueroa@gmail.com>.
} 
564 | D QUEIROZ et al.

\section{Conclusão}

A associação observada entre deficit de altura e menores níveis de retinol sérico em crianças de menor faixa etária mostra a maior vulnerabilidade desse grupo para sofrer deficits vitamínicos e de crescimento.

Termos de indexação: Crescimento. Deficiência de vitamina A. Estado nutricional. Vitamina A.

\section{A B S T R A C T}

\section{Objective}

This study assessed the relationship between anthropometric indices and the serum retinol level of children under age five years from the State of Paraíba.

\section{Methods}

This cross-sectional, population-based study measured the serum retinol level of 1,205 children to determine their vitamin A status and assessed their weight-for-height and height-for-age to determine their nutritional status.

\section{Results}

Stunted children aged 6 to 12 months had lower serum retinol levels than normal-height children $(p=0.02)$.

\section{Conclusion}

The association between stunting and low serum retinol levels in young children shows their greater vulnerability to vitamin and growth deficits.

Indexing terms: Growth. Vitamin A deficiency. Nutritional status. Vitamin A.

\section{N T R O D U ÇÃ O}

A Deficiência de Vitamina A (DVA) é um problema de saúde pública em várias regiões do mundo'. Estimativas indicam que 190 milhões de crianças pré-escolares têm DVA, o que corresponde a 33,3\% desse grupo em nível global. Nas Américas, o problema é considerado de saúde pública de grau moderado com prevalência de $15,6 \%^{2}$. No Brasil, a DVA é um problema particularmente importante na Região Nordeste ${ }^{3}$. Segundo a Pesquisa Nacional de Demografia e Saúde (PNDS), 17,4\% das crianças menores de cinco anos têm DVA, sendo as maiores prevalências observadas no Nordeste (19,0\%) e Sudeste (21,6\%) do País 4 .

A vitamina A é citada como um micronutriente de especial importância durante os períodos de proliferação e de rápida diferenciação celular, tais como a gestação, o período neonatal e a infância ${ }^{5,6}$. Além da importância no ciclo visual, a vitamina $A$ apresenta reconhecida relação com a integridade epitelial, com o sistema imunológico e com o crescimento 7 .
Estudo de metanálise sobre o efeito da suplementação com vitamina $A$ em crianças e adolescentes apontou ausência de impacto no incremento de estatura e peso ${ }^{8}$. Por sua vez, revisão sistemática de ensaios clínicos randomizados indicou resultado similar no crescimento linear de crianças $^{9}$. Entretanto, existem evidências sobre a influência da condição nutricional e/ou da idade na resposta a uma determinada intervenção nutricional ${ }^{10}$. Estudo de suplementação com vitamina A desenvolvido na Tanzânia, por exemplo, melhorou o crescimento em crianças com anemia ${ }^{11}$. No Brasil, em município do Estado de São Paulo, o benefício de um programa de suplementação alimentar foi maior em crianças com deficits nutricionais e em menores de um ano ${ }^{10}$. Em relação à vitamina $A$, sabe-se que maior efeito no crescimento é esperado nos casos de estoques funcionais esgotados e/ou quando sua deficiência resulta em um incremento da morbidade ${ }^{12}$, fenômenos particularmente importantes em crianças.

O objetivo do presente estudo foi avaliar a relação entre os índices antropométricos e os 
níveis de retinol sérico em crianças menores de cinco anos do Estado da Paraíba. Espera-se que os resultados apresentados gerem hipóteses para futuras pesquisas sobre a influência da DVA no crescimento infantil.

\section{MÉ TO D OS}

O presente estudo é parte dos resultados obtidos de uma pesquisa de base populacional desenvolvida em 2007 no Estado da Paraíba cujo objetivo foi avaliar a operacionalização do Programa Nacional de Suplementação de Vitamina A, bem como determinar a prevalência de DVA, anemia e desnutrição em pré-escolares ${ }^{13}$. O Protocolo de pesquisa foi aprovado pelo Comitê de Ética em Pesquisa da Universidade Estadual da Paraíba, sob o n 1128.0.133.000-05.

A referida pesquisa considerou uma amostra probabilística de 1324 crianças de 6 a 59 meses de idade de áreas urbanas do Estado da Paraíba. As crianças foram selecionadas aleatoriamente, segundo a técnica de amostragem do tipo dupla etapa, considerando-se o município ( $1^{\circ}$ conglomerado) e o setor censitário $\left(2^{\circ}\right.$ conglomerado). Assim, selecionaram-se nove municípios localizados nas regiões do sertão (Belém do Brejo do Cruz, Boa Ventura, Conceição, Malta, Patos, Pedra Branca e São José de Espinhares), do agreste (Campina Grande) e da zona da mata (João Pessoa) da Paraíba.

Os municípios de João Pessoa e Campina Grande foram previamente selecionados por apresentarem densidade populacional significativamente maior do que os demais municípios que compõem o Estado e por conta da localização (municípios em duas das três regiões bioclimáticas do Estado). Considerando que a distribuição do problema sob investigação possa ter a potencial interferência da localização cartográfica, a contribuição no espaço amostral dessas regiões, representadas pelos seus municípios de maior densidade populacional, imprime elementos que reforçam a representatividade da amostra. Os outros sete municípios foram selecionados por amos- tragem aleatória simples dentro da mesorregião do sertão, considerando a homogeneidade na densidade populacional entre os municípios.

Foram contemplados todos os pressupostos necessários para uma seleção probabilística das unidades amostrais, mediante o uso de uma tabela de números aleatórios na casualização dos setores censitários e domicílios, além de observado o limite máximo de 40 unidades amostrais por conglomerado (setor censitário). Foram convidados a participar do estudo crianças dos domicílios sorteados, com proporção de não resposta de aproximadamente $4 \%$, decorrente de recusas ou ausências. Adotou-se como critério de inclusão a residência fixa da criança no domicílio sorteado.

A colheita de sangue e a aferição das variáveis antropométricas foram realizadas entre 0 $1^{\circ}$ e $3^{\circ}$ dia útil subsequente à entrevista domiciliar destinada à coleta de informações socioeconômicas e demográficas. Os dados foram coletados em local próximo às residências, na sua maioria Unidades Básicas de Saúde da Família (UBSF). Na impossibilidade de utilizar as dependências das UBSF, a coleta foi realizada em clube de mães, igrejas ou associações de moradores.

Para realização dos exames laboratoriais, as amostras de sangue $(5 \mathrm{~mL})$ foram coletadas no início da manhã, sem a necessidade de jejum, por punção venosa periférica, com agulha e seringa descartáveis, e foram acondicionadas em tubos envolvidos por papel alumínio para proteger as amostras sanguíneas da luminosidade. Após retração do coágulo, o sangue foi centrifugado com velocidade de 3000rpm e alíquotas de soro foram acondicionadas em tubos ependorf âmbar para a determinação das concentrações de retinol sérico. As amostras provenientes dos municípios do sertão foram armazenadas e transportadas até os laboratórios de análises, mantendo-se a cadeia de frio. O período máximo de estocagem das amostras foi de três meses.

A determinação das concentrações de retinol sérico foi realizada por Cromatografia Líquida de Alta Eficiência, segundo a técnica proposta por Furr et al. ${ }^{14}$, no Centro de Investigação em 
Micronutrientes da Universidade Federal da Paraíba. As características do equipamento e laboratório utilizados para dosagem das concentrações de retinol sérico são descritas a seguir. Do equipamento: (i) Marca: Dionex; (ii) Modelo: Ultimate 3000; (iii) Tipo de coluna e detector: Alcclaim C 18, 5um 18å 4,6x250mm, Ultimate 3000 com Wavelength detector operando na região ultra-violeta a $325 \mathrm{~nm}$, column compartment guarda-nucleosil 18 - 20 $\mu \mathrm{L}$, Autosampler e Pump; (iv) Padrão: Acetato de retinol - sigma; (v) Tipo de saída de dados: Chromeleon 6.xx - Dionex; (vi) Retenção do pico: -3,6min; (vii) Fluxo de fase móvel: 1,5mL/min; (viii) Tipo de saída de dados: Chromeleon 6.xx - Dionex; (ix) Fluxo de fase móvel: 1,5mL/min; $(x)$ Fase móvel: Metanol. Do laboratório: (i) Espaço físico: Adequado para a análise de vitamina A (permite a semiobscuridade); (ii) Número de amostras realizadas/dia: 48 amostras em duplicata; (iii) Uso de padrão: Externo (acetato de retinol); (iv) Rotina controle de qualidade: Curva de calibração padrão (trimestral); (v) Determinação da acurácia: Externa; (vi) Determinação da precisão: pool; (vii) Análise em duplicata: $10 \%$ da rotina diária (amostra desprezada com diferença maior de 10\%).

As concentrações de retinol sérico foram categorizadas em níveis adequados $(\geq 1,05 \mu \mathrm{mol} /$ $\mathrm{L})$, níveis aceitáveis $(\geq 0,70 \mu \mathrm{mol} / \mathrm{L}$ e $<1,05 \mu \mathrm{mol} / \mathrm{L})$ e deficiência de vitamina $A(<0,70 \mu \mathrm{mol} / \mathrm{L})^{7}$.

As medidas de peso e altura foram tomadas por antropometristas, previamente treinados, seguindo recomendações internacionais ${ }^{15}$. Mensuraram-se as crianças usando vestuário mínimo, sem adornos na cabeça e sem calçados. Todas as medidas foram tomadas em duplicata, calculando-se a média das duas medidas. O peso das crianças menores de 24 meses foi obtido pela pesagem da mãe com a criança no colo, subtraindo-se posteriormente o peso da mãe. O comprimento das crianças menores de 24 meses foi obtido em decúbito dorsal. As crianças com 24 meses ou mais tiveram sua estatura medida em pé. Para a obtenção do peso utilizou-se balança digital eletrônica portátil (Tanita), com capacidade para $150,0 \mathrm{~kg}$ e sensibilidade de 0,1 kg. Para a obtenção do comprimento, utilizou-se infantômetro de madeira manufaturado, com escala 0-100 cm e precisão de $0,5 \mathrm{~cm}$. Para a obtenção da estatura, utilizou-se estadiômetro portátil (Seca), com escala de $0-220 \mathrm{~cm}$ e precisão de $0,1 \mathrm{~cm}$.

O estado nutricional das crianças foi expresso em escore-Z de acordo com o padrão de referência do Multicentre Growth Study, atualmente recomendado pela Organização Mundial da Saúde ${ }^{16}$. Os cálculos foram realizados com ajuda do programa World Health Organization (WHO) Anthro 2005 versão beta (WHO, Genebra, Suíça). Para a definição de deficit de altura, foi adotado o índice altura para idade (altura/idade) <-2 escore-Z. Para a definição do deficit de peso, foi adotado o índice peso para altura (peso/altura) $<-2$ escore- $Z^{15}$.

Os dados foram digitados e analisados por meio do software Epi Info v.6.04b (WHO/Centers for Disease Control and Prevention, Atlanta, GE, USA); a digitação foi em dupla entrada; e o aplicativo Validate foi utilizado para analisar a consistência.

Consideraram-se como variáveis dependentes, os deficits de altura e peso, e como variável de exposição, os níveis de retinol sérico. Foi calculada a Razão de Chances a fim de analisar a associação e a chance de deficit de altura e deficit de peso nas crianças com níveis séricos de retinol não adequados, verificando-se o Intervalo de Confiança de 95\% (IC95\%). Posteriormente, para verificar diferença estatística significante nas médias de retinol sérico entre as crianças com e sem deficit de altura, bem como entre as crianças com e sem deficit de peso, foi utilizado o teste $t$ Student. Para as análises, as crianças foram categorizadas em diferentes faixas etárias. A significância estatística das associações foi estabelecida com base no $p$ valor $<0,05$.

\section{RESULTADOS}

Foram avaliadas 1205 crianças com idade entre 6 e 59 meses, o que correspondeu a 9\% de 
perdas da amostra inicialmente calculada. As perdas ocorreram em função de recusa à participação, material biológico insuficiente para as análises bioquímicas ou não realização da antropometria.

A média de retinol das crianças foi de 0,90 $\mu \mathrm{mol} / \mathrm{L}(\mathrm{DP}=0,24 \mu \mathrm{mol} / \mathrm{L})$ sem diferença estatística entre as faixas etárias ( $6 \mid-12$ meses; 12 |- 24 meses; 24 |- 36 meses; 36 - 48 meses; 48 |- 60 meses) $(p=0,44)$ (dado não publicado em Tabela). Em relação aos deficits nutricionais, observou-se que $7,22 \%$ das crianças apresentaram deficit de estatura, e 1,08\%, deficit de peso (Tabela 1).

As crianças com DVA e aquelas com concentrações de retinol sérico em níveis aceitáveis apresentaram maior chance de deficit de altura quando comparadas às crianças com níveis ade- quados. Para o deficit de peso, não foi encontrada associação (Tabela 2).

Na Tabela 3, é possível observar que as crianças com deficit de altura na faixa etária de 6 |- 12 meses apresentaram retinolemia significa-

Tabela 1. Distribuição de crianças de 6 a 59 meses de idade segundo o estado nutricional antropométrico. Paraíba, 2007.

\begin{tabular}{lcr}
\hline Índices antropométricos & $\mathrm{n}$ & $\%$ \\
\hline Altura/ldade (escore-Z) & & \\
Sem deficit de altura $(\geq-2)$ & 1118 & 92,78 \\
Com deficit de altura $(<-2)$ & 87 & 7,22 \\
Peso/Altura (escore-Z) & & \\
Sem deficit de peso $(\geq-2)$ & 1192 & 98,92 \\
Com deficit de peso $(<-2)$ & 13 & 1,08 \\
\hline
\end{tabular}

Tabela 2. Associação entre níveis séricos de retinol e estado nutricional antropométrico de crianças de 6 a 59 meses de idade. Paraíba, 2007.

\begin{tabular}{lcccc}
\hline \multirow{2}{*}{ Níveis séricos de retinol $(\mu \mathrm{mol} / \mathrm{L})$} & \multicolumn{2}{c}{ Deficit de altura $(\mathrm{A} / \mathrm{I}<-2$ escore-Z) } & & \multicolumn{2}{c}{ Deficit de altura (P/A <-2 escore-Z) } \\
\cline { 2 - 3 } & Razão de chances (IC95\%) & $p$ & Razão de chances (IC95\%) \\
\hline Adequados $(\geq 1,05)$ & 1 & & 1 \\
Aceitáveis $(0,70 \mid-1,05)$ & $2,878(1,401-5,912)$ & 0,001 & $0,754(0,179-3,176)$ \\
Deficiência de vitamina A $(<0,70)$ & $3,129(1,427-6,861)$ & 0,002 & & $1,852(0,438-7,825)$ \\
\hline
\end{tabular}

Nota: All: Altura para Idade; P/A: Peso para Altura; IC95\%: Intervalo de Confiança de $95 \%$.

Tabela 3. Distribuição das médias de retinol sérico (ìmol/L) de crianças de 6 a 59 meses de idade por faixa etária e estado nutricional antropométrico. Paraíba, 2007.

\begin{tabular}{|c|c|c|c|c|c|c|c|c|c|c|c|c|}
\hline \multirow{2}{*}{ Faixa etária (meses) } & \multicolumn{2}{|c|}{$\begin{array}{l}\text { Sem deficit de } \\
\text { altura }(A / l \geq-2 Z)\end{array}$} & & \multicolumn{2}{|c|}{$\begin{array}{c}\text { Com deficit de } \\
\text { altura }(\mathrm{A} / \mathrm{l}<-2 \mathrm{Z})\end{array}$} & \multicolumn{2}{|c|}{$\begin{array}{l}\text { Sem deficit de } \\
\text { altura }(A / l \geq-2 Z)\end{array}$} & & \multicolumn{2}{|c|}{$\begin{array}{c}\text { Com deficit de } \\
\text { altura }(\mathrm{A} / \mathrm{l}<-2 \mathrm{Z})\end{array}$} & \multicolumn{2}{|c|}{ Total } \\
\hline & $M$ & DP & & $M$ & DP & $M$ & DP & & $M$ & DP & $n$ & $\%$ \\
\hline \multirow[t]{2}{*}{$6 \mid-12$} & \multirow{2}{*}{\multicolumn{2}{|c|}{$0,92 \pm 0,23$}} & & \multicolumn{2}{|c|}{$0,76 \pm 0,14$} & \multirow{2}{*}{\multicolumn{2}{|c|}{$0,91 \pm 0,23$}} & & \multirow{2}{*}{\multicolumn{2}{|c|}{$1,10 \pm 0,00^{* *}$}} & 125 & 10,38 \\
\hline & & & $p^{*}=0,02$ & & & & & $p=0,40$ & & & & \\
\hline \multirow[t]{2}{*}{$12 \mid-24$} & \multirow{2}{*}{\multicolumn{2}{|c|}{$0,90 \pm 0,24$}} & & \multicolumn{2}{|c|}{$0,83 \pm 0,19$} & \multicolumn{2}{|c|}{$0,89 \pm 0,24$} & & \multirow{2}{*}{\multicolumn{2}{|c|}{$0,83 \pm 0,06$}} & 279 & 23,15 \\
\hline & & & $p=0,16$ & & & & & $p=0,71$ & & & & \\
\hline \multirow[t]{2}{*}{$24 \mid-36$} & \multirow{2}{*}{\multicolumn{2}{|c|}{$0,87 \pm 0,27$}} & & \multirow{2}{*}{\multicolumn{2}{|c|}{$0,96 \pm 0,20$}} & \multirow{2}{*}{\multicolumn{2}{|c|}{$0,88 \pm 0,24$}} & & \multirow{2}{*}{\multicolumn{2}{|c|}{$0,50 \pm 0,14$}} & 246 & 20,41 \\
\hline & & & $p=0,23$ & & & & & $p=0,03$ & & & & \\
\hline \multirow[t]{2}{*}{$36 \mid-48$} & \multirow{2}{*}{\multicolumn{2}{|c|}{$0,90 \pm 0,23$}} & & \multirow{2}{*}{\multicolumn{2}{|c|}{$0,79 \pm 0,17$}} & \multirow{2}{*}{\multicolumn{2}{|c|}{$0,90 \pm 0,23$}} & & \multirow{2}{*}{\multicolumn{2}{|c|}{$0,80 \pm 0,28$}} & 281 & 23,32 \\
\hline & & & $p=0,05$ & & & & & $p=0,55$ & & & & \\
\hline \multirow[t]{2}{*}{$48 \mid-60$} & \multirow{2}{*}{\multicolumn{2}{|c|}{$0,92 \pm 0,25$}} & & \multirow{2}{*}{\multicolumn{2}{|c|}{$0,81 \pm 0,21$}} & \multirow{2}{*}{\multicolumn{2}{|c|}{$0,92 \pm 0,25$}} & & \multirow{2}{*}{\multicolumn{2}{|c|}{$0,87 \pm 0,26$}} & 274 & 22,74 \\
\hline & & & $p=0,12$ & & & & & $p=0,63$ & & & & \\
\hline \multirow[t]{2}{*}{ Total } & \multirow{2}{*}{\multicolumn{2}{|c|}{$0,90 \pm 0,24$}} & & \multicolumn{2}{|c|}{$0,83 \pm 0,18$} & \multirow{2}{*}{\multicolumn{2}{|c|}{$0,90 \pm 0,25$}} & & \multirow{2}{*}{\multicolumn{2}{|c|}{$0,81 \pm 0,27$}} & 1205 & 100 \\
\hline & & & $p<0,001$ & & & & & $p<0,21$ & & & & \\
\hline
\end{tabular}

Nota: ${ }^{*}$ Teste $t$ Student; ${ }^{* *}$ Apenas uma criança apresentou deficit de peso nessa faixa etária.

All: Altura para Idade; P/A: Peso para Altura. M: Média; DP: Desvio-Padrão. 
568 | D QUEIROZ et al.

tivamente inferior quando comparadas às crianças sem deficit de altura $(p=0,02)$. Além disso, as crianças com deficit de peso, na faixa etária de 24 |- 36 meses, apresentaram concentrações de retinol inferiores quando comparadas às crianças sem deficit de peso $(p=0,03)$. Considerando a população total, a significância estatística manteve-se apenas para o deficit de altura $(p<0,01)$.

\section{I S C U S S Ã O}

Os resultados do presente estudo apontaram a influência da faixa etária na relação entre o estado nutricional de vitamina A e o antropométrico. Apesar de bem documentada a importância da vitamina A no crescimento ${ }^{17}$, estudos sistematizados questionam o desempenho da suplementação com vitamina A nos indicadores de peso e altura ${ }^{8,9}$. Entretanto, observações e experimentos mostram resultados diversos. Trabalhos como os de Khandait et al. ${ }^{18}$, Khatib \& Elmadfa ${ }^{19}$ e Maslova et al. ${ }^{20}$ descreveram a DVA associada à desnutrição. Resultados contrários foram registrados por Assis et al. ${ }^{21}$, Castejón et al. ${ }^{22}$, Hautvast et al. ${ }^{23}$ e Lartey et al. ${ }^{24}$. Entretanto, o efeito da suplementação com vitamina A, anteriormente relatado, é questionável devido aos resultados de experimentos mais recentes que desvendam impacto positivo $25-27$.

Questionam-se, assim, os fatores aos quais essas diferenças podem ser atribuídas. Hadi et al. ${ }^{28}$ destacam que diferenças ambientais, nas concentrações de retinol e de outros nutrientes, assim como mudanças nas demandas de nutrientes associadas à idade e a doenças infecciosas são fatores que podem influenciar os resultados relacionados à associação entre o estado nutricional de vitamina A e antropométrico. Importa, também, reconhecendo o crescimento linear e ponderal como entidades diferentes, considerar as características próprias desses desfechos e a magnitude do deficit no crescimento linear e ponderal nos grupos de interesse. O deficit de altura resulta de um processo caracterizado pela redução da frequência de eventos de crescimento, que pode ter início na vida uterina, e não, neces- sariamente, refletir o momento atual. Por sua vez, o deficit ponderal reflete mais as condições atuais, tendo como causas diretas a ingestão inadequada (quantidade e qualidade), e a alta incidência de doenças diarreicas ${ }^{29}$. Assim, além da questão ambiental e das demandas nutricionais, a temporalidade constitui um fator relevante na relação causal entre o estado nutricional de vitamina A e antropométrico.

Como argumento das razões anteriormente ilustradas, pode-se invocar a variedade de achados em diferentes trabalhos. Estudo desenvolvido na China ${ }^{25}$ mostrou que tanto a fortificação com múltiplos micronutrientes quanto com vitamina $A$ e ferro incrementaram a altura de crianças pré-escolares, sendo significativamente maior o produzido pela combinação múltipla de micronutrientes. Porém, em relação ao peso, o trabalho trouxe um resultado inesperado ao constatar incremento de peso/altura somente nas crianças que foram intervindas exclusivamente com vitamina A. Na Indonésia ${ }^{28}$, altas doses de vitamina $A$ foram responsáveis pela melhora no crescimento linear de crianças entre 4 e 48 meses de idade, sendo o efeito maior nas crianças com níveis séricos baixos de vitamina $A$, maiores de 24 meses e não amamentadas. Estudo realizado no Brasil $^{26}$ constatou que a vitamina $A$ pode beneficiar o estado nutricional por efeito da apolipoproteína E4. Outro estudo chinês ${ }^{27}$ indicou que, após a intervenção, tanto a altura quanto o peso de criancas pré-escolares melhoraram com diferentes esquemas de doses de vitamina $A$, sem diferença condicionada pela dose. Trabalho de cunho prospectivo randomizado com criancas brasileiras ${ }^{30}$ revelou que, apesar do tratamento com vitamina A mostrar-se eficaz no parasitismo, esse efeito nao atingiu o peso nem a altura. Em Gana ${ }^{24}$, a associação entre as concentrações de retinol e o crescimento em crianças de 1 a 18 meses amamentadas não foi encontrada. Na Colômbia ${ }^{20}$, o deficit ponderal mostrou-se associado à deficiencia de vitamina A, contrariamente ao resultado mostrado para o deficit de altura. 


\section{O N CLUS Ã O}

Os estudos observacionais e experimentais, pautados na hipótese de relação causal entre a vitamina $\mathrm{A}$ e o crescimento pondero-estatural, mostram resultados diversos, ora com associação/impacto, ora sem. Dessa forma: i) os dados existentes parecem ser ainda insuficientes para a conclusão sobre o papel exato da deficiência de vitamina A no crescimento ao nível populacional; ou ii) parece que índices antropométricos e retinolemia podem estar associados quando fatores ambientais e de demanda biológica são contribuintes. Ao considerar os resultados deste estudo, a faixa etária da criança parece ser um fator importante, com comportamento para o crescimento linear diferente ao do ponderal.

Isto posto, a vitamina A, por ser lipossolúvel, pode ser armazenada em grandes quantidades no organismo humano e possui mecanismo de eliminação lento. Dessa forma, os efeitos da carência alimentar de vitamina A só se manifestam fisiologicamente em período relativamente longo (vários meses), o que faz do organismo humano a possibilidade de prescindir da ingestão diária de compostos de retinóis. Essa característica é importante na hora de considerar a plausibilidade biológica das variações relacionadas aos efeitos da deficiência em função da fisiologia e metabolismo do organismo, bem como da exigência, capacidade de absorção e aproveitamento. Outro aspecto importante é que alterações no crescimento acontecem na influência de deficiências simultâneas de nutrientes, sendo dificilmente a deficiência de vitamina A um fenômeno isolado, principalmente entre crianças. A maior vulnerabilidade das crianças menores de 12 meses, devido a situações como abandono precoce do aleitamento materno, monotonia alimentar e maior propensão a doenças infectoparasitárias, produz aumento das demandas metabólicas que interferem no processo de armazenamento, transporte e utilização da vitamina $A$, o que pode justificar a influência exercida pela faixa etária na relação retinolemia e crescimento.

\section{A GRADECIMENTOS}

Ao Conselho Nacional de Desenvolvimento Científico e Tecnológico pelo financiamento concedido; aos técnicos do laboratório Cimicron pelo rigor nas análises bioquímicas e pela boa receptividade dada à nossa equipe; à Secretaria de Saúde do Estado da Paraíba, na pessoa da Dra Eliane Gadelha, e demais secretarias municipais das cidades incluídas no estudo, cujo apoio logístico foi fundamental para o êxito do estudo; à equipe de entrevistadores de campo e antropometristas, em sua maioria alunos da Universidade Estadual da Paraíba, pela colaboração inestimável na coleta de dados.

\section{COLABORADORES}

D QUEIROZ participou do desenvolvimento do protocolo do estudo; da coleta, análise e interpretação dos dados; e da redação do artigo. AA PAIVA participou do desenvolvimento do protocolo de estudo; da interpretação dos dados; e da redação do artigo. JSFA GAMA participou da coleta, análise e interpretação dos dados; e da revisão do artigo. ZN LIMA realizou as análises bioquímicas e participou da interpretação dos resultados e revisão do artigo. D FIGUEROA PEDRAZA participou da análise e interpretação dos dados; redação e revisão final do artigo.

\section{REFERÊ NCIAS}

1. Milagres RCRM, Nunes LC, Sant'Ana HMP. A deficiência de vitamina $A$ em crianças no Brasil e no mundo. Ciênc Saúde Coletiva. 2007; 12(5):1253-66.

2. World Health Organization. Global prevalence of vitamin A deficiency in populations at risk 1995-2005: WHO global database on vitamin A deficiency. Geneva: WHO; 2009.

3. Geraldo RRC, Paiva SAR, Pitas AMCS, Godoy I, Campana AO. Distribuição da hipovitaminose A no Brasil nas últimas quatro décadas: ingestão alimentar, sinais clínicos e dados bioquímicos. Rev Nutr. 2003; 16(4):443-60. doi: 10.1590/S1415-527 32003000400008.

4. Brasil. Ministério da Saúde. Pesquisa nacional sobre demografia e saúde da criança e da mulher. Brasília: MS; 2008.

5. Valente SLS, Thiapó AP, Souza GG, Saunders C, Ramalho A. Micronutrientes na gestação e lactação. Rev Bras Saúde Matern Infant. 2007; 7(3):237-44.

6. Brasil. Ministério da Saúde. Carências de micronutrientes. Brasília: MS; 2007. 
570 | D QUEIROZ et al.

7. West Jr KP. Extent of vitamin A deficiency among preschool children and women of reproductive age. J Nutr. 2002; 132(9):2857S-66.

8. Ramakrishnan U, Aburto N, McCabe G, Martorell R. Multimicronutrient interventions but not vitamin a or iron interventions alone improve child growth: Results of 3 meta-analyses. J Nutr. 2004; 134(10): 2592-602.

9. Bhandari N, Bahl R, Taneja S. Effect of micronutrient supplementation on linear growth of children. $\mathrm{Br}$ J Nutr. 2001; 85(Suppl 2):131S-7.

10. Carvalho LG, Saldiva SRDM, Rosa TEC, Lei DLM. Evolução do estado nutricional de crianças submetidas a um programa de suplementação alimentar em município do Estado de São Paulo. Rev Nutr. 2009; 22(2):207-17. doi: 10.1590/S1415-527320 09000200003.

11. Mwanri L, Worsley A, Ryan P, Masika J. Supplemental vitamin A improves anemia and growth in anemic school children in Tanzania. J Nutr. 2000; 130(11): 2691-96.

12. Figueroa Pedraza D, Queiroz D. Micronutrientes no crescimento e desenvolvimento infantil. RBCDH. 2011; 21(1):155-70.

13. Paiva AA, Cagliari MPP, Queiroz D, Souto RA, Brito VRS, França ISX. Programa nacional de suplementação de Vitamina A no Estado da Paraíba: uma análise a partir do relato de profissionais da Equipe de Saúde da Família. Epidemiol Serv Saúde. 2011; 20(3):373-83.

14. Furr HC, Tanumiharjo S, Olson JA. Training manual for assessing vitamin A status by use the modified relative dose response assays. Ames: lowa State University; 1992.

15. World Health Organization. Physical status: The use and interpretation of anthropometry. Geneva: WHO; 1995. WHO Technical Report Series, 854.

16. World Health Organization. Length/height-for- age, weight-for-age, weight-for-length, weight-forheight and body mass index-for-age: Methods and development. Geneva: WHO; 2006.

17. Singh M. Role of micronutrients for physical growth and mental development. Indian J Pediatr. 2004; 71(1):59-62.

18. Khandait DW, Vasudeo ND, Zodpey SP, Kumbhalkar DT, Koram MR. Subclinical vitamin A deficiency in undersix children in Nagpur, India. Southeast Asian J Trop Med Public Health. 1998; 29(2):289-92.

19. Khatib IMD, Elmadfa I. High prevalence rates of anemia, vitamin A deficiency and stunting imperil the health status of Bedouin schoolchildren in North Badia, Jordan. Ann Nutr Metab. 2009; 55(4):358-67.

20. Maslova E, Mora-Plazas M, Forero Y, López-Arana S, Baylin A, Villamor E. Are vitamin A and iron deficiencies re-emerging in urban Latin America? A survey of schoolchildren in Bogota, Colombia. Food Nutr Bull. 2009; 30(2):103-11.
21. Assis AMO, Prado MS, Freitas MCS, Cruz MM. Deficiência de vitamina A e desnutrição energético-protéica em crianças de localidades do semi-árido baiano. Rev Nutr. 1997; 10(1):70-8.

22. Castejón HV, Ortega P, Díaz ME, Amaya D, Gomez $G$, Ramos M, et al. Prevalence of sub-clinical vitamin A deficiency and malnutrition in slum children in Maraicabo-Venezuela. Arch Latinoam Nutr. 2001; 51(1):25-32.

23. Hautvast JL, Tolboom JJM, Kafwembe EM, Musonda RM, Mwanakasal V, Van Staveren WA, et al. Severe linear growth retardation in rural Zambian children: The influence of biological variables. Am J Clin Nutr. 2000; 71:550-9.

24. Lartey A, Manu A, Brown KH, Peerson JM, Dewey KG. Predictors of growth from 1 to 18 months among breast-fed Ghanaian infants. Eur J Clin Nutr. 2000; 54(1):41-9.

25. Chen K, Li TY, Chen L, Qu P, Liu YX. Effects of vitamin $A$, vitamin $A$ plus iron and multiple micronutrient-fortified seasoning powder on preschool children in a suburb of Chongqing, China. J Nutr Sci Vitaminol. 2008; 54(6):440-7.

26. Mitter SS, Oriá RB, Kvalsund MP, Pamplona P, Joventino ES, Mota RMS, et al. Apolipoprotein E4 influences growth and cognitive responses to micronutrient supplementation in shantytown children from Northeast Brazil. Clinics. 2012; 67(1):11-8

27. Zhang X, Ke Cheng K, Qu P, Liu YX, Li TY. Effect of biscuits fortified with different doses of vitamin $A$ on indices of vitamin A status, haemoglobin and physical growth levels of preschool children in Chongqing. Public Health Nutr. 2010; 13(9):1462-71.

28. Hadi H, Stoltzfus RJ, Dibley MJ, Moulton LH, West Jr KP, Kjolhede, et al. Vitamin A supplementation selectively improves the linear growth of Indonesian preschool children: Results from a randomized controlled trial. Am J Clin Nutr. 2000; 71(2):507-13.

29. Borges CVD, Veiga APB, Barroso GS, de Jesus EFO, Serpa RFB, Moreira S, et al. Associação entre concentrações séricas de minerais, índices antropométricos e ocorrência de diarreia entre crianças de baixa renda da região metropolitana do Rio de Janeiro. Rev Nutr. 2007; 20(2):159-69. doi: 10.15 90/S1415-52732007000200005.

30. Lima AAM, Soares AM, Lima NL, Mota RMS, Maciel BLL, Kvalsund MP, et al. Vitamin A supplementation effects on intestinal barrier function, growth, total parasitic, and specific Giardia spp. infections in Brazilian children: A prospective randomized, doubleblind, placebocontrolled trial. J Pediatr Gastroenterol Nutr. 2010; 50(3):309-15.

Recebido em: 29/9/2012

Versão final em: 27/5/2013

Aprovado em: 1/7/2013 